\title{
Scheduling for Source Relaying with Packet Aggregation in Industrial Wireless Networks
}

\begin{abstract}
The interest in wireless communication systems for industrial applications has grown significantly over the last years. More flexible, easier to install and maintain wireless networks present a promising alternative to the currently used wired systems. However, reliability and timeliness requirements at present met by wired networks need also be fulfilled by wireless solutions. Packet errors introduced when packets travel through wireless channels imply a significant challenge to fulfill these requirements. Relaying has been recognised to improve reliability in industrial wireless networks without causing additional delay. Further, recent results have shown that relaying combined with packet aggregation significantly outperforms simple relaying. However, it is not always costefficient to introduce additional relay nodes into an industrial network and hence, in this paper, we propose to use a combination of relaying and packet aggregation at the source nodes. The results show that when relaying and aggregation are used at the source nodes, the transmission schedule plays a crucial role. A schedule adapting to the varying channel conditions improves the performance substantially. By carefully choosing which packet to aggregate, even further improvements can be achieved.
\end{abstract}

\section{INTRODUCTION}

Great benefits can be achieved by replacing currently used wired networks with wireless systems. Not requiring many meters of wires makes the tasks of installation and maintenance of a network much easier. Additionally, wireless networks give an opportunity to enable new application areas, such as e.g. controlling of moving robots. Moreover, wireless solutions are more flexible and their usage can lead to significant cost reductions. Several new standards for Industrial Wireless Sensor Networks (IWSNs) like e.g. WirelessHART [1, 2], WIA-PA [3], ISA100.11a [4] and IEEE 802.15.4e [5] were developed and investigated over the past years [6-10]. However, in wireless systems, transmitted packets are affected by the channels they propagate through and thus the number of packet errors in wireless networks is much higher compared to their wired counterparts. There are several traditional techniques, such as e.g. retransmissions and error control codes, which can be used to reduce the number of errors in wireless systems. However, error control codes only work for channels with bursts of errors shorter than the correction capability of the code and retransmissions can, in some situations, lead to extensive delays and thus deadline misses. Too many missed deadlines or packet errors in industrial systems will lead to financial losses due to damage of expensive equipment, stop of production or, depending on the application, even danger to humans. Thus, to be suitable for industrial use, wireless systems need robust techniques to deal with errors.

An interesting approach to improve the performance of wireless networks is exploitation of spatial diversity, e.g. introducing relaying $[11,12]$. In relaying schemes, there are a number of intermediate nodes set to overhear source transmissions and aid these transmissions by forwarding the overheard packets to their destinations. It has previously been established that relay nodes can improve the achievable reliability for deadline-constrained data traffic in industrial networks substantially [13-16]. However, placing additional nodes for the sole purpose of relaying might not always be desirable or cost-effective.

In this paper we consider a TDMA-based industrial wireless system where all source nodes function as relayers and hence all nodes are instructed (at the expense of additional energy consumption) to overhear data sent by other source nodes. The considered TDMA scheme is comparable to WirelessHART, which we also use as a reference system. Every source node is assigned a time slot to transmit in. In its assigned time slot each node uses the packet aggregation mechanism from [17] to transmit its own data packet plus the data from one of the packets it has overheard from sources with earlier time slots. The term packet aggregation [17] stands for concatenation of payloads from more than one data packet into a larger one sharing the same header. Packet aggregation in industrial networks exploits the fact that most packets in IWSNs tend to be small compared to the size of a TDMA time slot typically encountered in e.g., WirelessHART. It was shown in [17] that packet aggregation can be introduced into WirelessHART with only minor changes in the standard and its introduction, depending on the network topology, allows up to $50 \%$ energy saving. In this paper we show that packet aggregation, when combined with source-based relaying, can furthermore help to save time slots that otherwise would be required for retransmissions. As with any other packet forwarding technique, a specific scheduling scheme ensuring deterministic end-to-end delay is required to allocate the time slots [18]. Since every node is allowed to do packet aggregation, it is important for the overall performance of the system that most nodes actually are able to use the opportunity to aggregate. The probability that all nodes, except the first one, have overheard at least one other packet to aggregate depends on the order in which the sources transmit their data. In addition, a source node having overheard multiple packets from sources in earlier time slots has to decide which of these packets it should aggregate with its own. The main goal of this paper is to study decision schemes for these two system aspects: the transmission order of the source nodes and the local choice of which packet to aggregate, with the goal of improving the likelihood of having all packets received at the destination before their deadlines. Several source scheduling schemes both with and without channel adaptation are studied as well as several different packet aggregation strategies. The current paper continues the work in [19] by the authors and presents results for a wider range of simulation scenarios considering several performance indicators. The results show that packet aggregation by itself leads to significant performance improvements. When the source transmission schedule is adjusted to the channel conditions, the gain grows and even further improvement can be achieved by making the choice of packets to aggregate aware of current source transmission schedule as well as of the actions of other sources. 
The remainder of the paper is structured as follows: in Section II the system model is described, while Section III contains the evaluated relay schemes. Simulation results are given in Section IV and finally Section V concludes the paper.

\section{SYSTEM MODEL}

In this work we consider a typical industrial network consisting of a number of sensors, measuring temperature, pressure, humidity, etc. and sending their readings to a central controller or, as in e.g. WirelessHART, a gateway that forwards the data to a programmable logic controller (PLC) using a wired bus. We study a setup with $N$ sensor nodes numbered from 0 to $N-1$ and one controller, which is denoted as node $N$; we consider only uplink data transmission in a TDMA-based IWSN similar to what is used in WirelessHART, ISA100.11a, or IEEE 802.15.4 LLDN. The TDMA protocol sub-divides time into consecutive superframes (also referred to as transmission rounds), and each superframe is in turn sub-divided into a fixed number of time slots. One time slot, e.g. $10 \mathrm{~ms}$ in WirelessHART, is sufficient to transmit a data packet of maximum size as well as to send and receive an acknowledgement. As separate relay nodes typically are not cost-efficient in industrial networks, we suggest to use existing source nodes for relaying. This requires extra energy on the source nodes due to the need to listen more often to overhear packets sent by other sources and aggregating them with their own one. However, industrial sensors are often attached to a permanent power supply, and relaying in itself may reduce the number of retransmissions required and thus result in only a minor increase in energy. Furthermore, reliability and timeliness are the main requirements in IWSNs and thus additional energy may be spent to guarantee the required performance level.

We look at a network where all the nodes are randomly placed within the deployment area. Between each pair of nodes, a separate channel with a given packet error rate exists which is stochastically independent of all other channels and symmetric in both directions. All channels remain constant during the transmission of a long packet. The packet error rates for all possible channels between pairs of nodes are collected into a quadratic and symmetric channel matrix $\mathbf{C}$ of dimension $N+1$; we denote the entries of $\mathbf{C}$ by $c_{i . j}$, which refers to the packet error rate on the channel between stations $i$ and $j$. This channel model considers long-term average packet loss rates between stations and covers a wide range of channels. In particular, this model covers frequency-hopping channels (used in WirelessHART) since in the long-term, such a channel is essentially dominated by the path loss between stations, since the frequency-hopping only eliminates the effects of the short-term fading. For similar reasons, the model also covers channels with fast fading, as again such channels are dominated in the long run by the path loss, and the short-term variation is averaged out. Furthermore, please note that these assumptions about the channel are commensurate with the operation of WirelessHART, where the network manager collects long-term packet loss rates from individual stations as a basis for constructing a schedule.

One important limitation of the IEEE 802.15.4 physical layer is that packets can have a maximum length of 133 bytes and the MAC packets are restricted to 127 bytes. The data packets used in IWSNs are relatively small and thus it is possible to apply packet aggregation, i.e., concatenate two or more source packets into one larger packet, with only one single header. More specifically, we use the data aggregation scheme proposed in [17] for WirelessHART. This scheme suggests to aggregate the packets directed to the same destination. Each un-aggregated packet has a PHY header of 6 bytes in total ( 4 bytes preamble, 1 byte startframe-delimiter and 1 byte as byte-count field), a MAC header and trailer of 16 bytes in total (10 bytes header, 4 bytes for a linklevel message integrity check field, and 2 bytes for an end-offrame CRC checksum) followed by a network layer header of 16 bytes and the actual payload of $x$ bytes, resulting in a total unaggregated packet length of $6+16+16+x$ bytes. In an aggregated packet, the MAC layer header (which comes after the PHY header) is followed by 8 bytes constituting the common network layer header. Following this, if $\alpha$ different source packets are aggregated, then for each of the aggregated packets, a private network layer header of 9 bytes is needed, followed by the payload of $x$ bytes. So, an aggregated packet has a total length of $6+16+8+\alpha(9+x)$ bytes, assuming all data packets have the same payload length $x$. Thus, while still fulfilling the requirement of the maximum packet size of 133 bytes, it is for example possible to aggregate three 62 bytes long or two 80 bytes long packets, which will result in 129 or 133 bytes long aggregated packets, respectively. This aggregation scheme was investigated together with relaying in [20] and it was shown that relaying combined with packet aggregation significantly outperforms simple relaying and thus, a similar scheme is adopted in this work. It was further shown that under an assumption of 62 bytes long source packets, three source packet payloads can be concatenated in an aggregated packet. To maintain a reasonable complexity, we assume all the packets to be of the same size and let each source aggregate its own packet with only one other overheard packet. Longer packets may have higher packet error rates, however for simulation simplicity we assume that all packets experience the same packet error rate. This is a reasonable assumption for packets with a relatively small (compared to other wireless technologies) maximal size of just 133 bytes in total.

We make one further assumption. In particular we assume that the central controller has the opportunity to transmit configuration data to all nodes at the start of each superframe. In technologies like IEEE 802.15.4 LLDN, this assumption is naturally fulfilled by the presence of beacons (allowed to also include additional payload) transmitted at the start of each superframe, whereas in technologies like WirelessHART (where such beacons are not mandatory) one could schedule regular slots for this purpose. This assumption is particularly important for the adaptive scheduling scheme introduced below, where this opportunity is used by the central controller to periodically send an updated schedule to the sensors.

\section{PROPOSED SCHEMES}

All source packets are generated at the beginning of every superframe and all packets are assumed to have the same deadline, equal to the superframe length. Every source node is given one time slot in the superframe, which makes the superframe $\mathrm{N}-1$ slots long. Following most of the TDMA-based standards 
for industrial applications, the schedule is known to all the nodes in the beginning of each superframe and every time slot is pre-allocated to a specific sender-receiver pair. However, the sender is given the flexibility to choose what to send in its allocated slot. Thus, we propose that all source nodes overhear all packets sent in all slots preceding their own, keep these packets in their memory and when the time comes aggregate one of the overheard packets with their own transmission. When a source has overheard more than one packet from earlier sources, it has to select one of them. The overall goal of the work is to find a good TDMA schedule and a good packet selection strategy that maximizes the probability for the controller to receive all source packets before the deadline.

\section{1) Evaluated transmission schedules}

We compare four different TDMA scheduling options:

- Fixed schedule, the transmission order of sources within a superframe is chosen without further consideration as $0,1,2, \ldots, N-1$, and never changes.

- Random schedule, the schedule is different for every superframe and the order in which the sources transmit is chosen randomly each time.

- Round-robin schedule, the transmission order is changed in round-robin manner, i.e. if in the first superfame the schedule starts with node 0 and ends with node $N-1$, for the next superframe it will be node 1 that starts transmissions and node 0 which ends them.

- Adaptive schedule, the controller maintains estimates of the packet error rates between itself and each source (by continuously monitoring the reception of packets arriving from the sources and updating a simple moving-average type of estimator), and then schedules the sources in order of increasing channel quality, i.e. the "worst" source will transmit first. The schedule for the next superframe is calculated based on the updated channel estimations from the previous superframe. A similar schedule, but assuming perfect channel information available in all nodes, was used in [21] and showed good performance in terms of stable throughput regions and average delay.

\section{2) Evaluated algorithms for packet selection}

We consider five different strategies for how to select a good packet to aggregate in case of several overheard packets. We denote the subset of previously overheard source packets by source $i$ as $n_{i}$. Note that $n_{i}$ only contains the subset of packets which have not yet been correctly received by the destination. To keep track of which packets were successfully received by the destination, all the nodes listen to the feedback information, which the controller sends at the end of every time slot. Short feedback messages, specifying if a packet was received and which source packet was aggregated, are assumed to always be delivered (this assumption has been made for simplicity, but is actually achievable as the ACK packets are usually short and the central controller typically transmits its feedback with a high transmit power). However, for completion, we will discuss a few results addressing the case of unreliable feedback below. The following algorithms are considered:

- No aggregation scheme (NA), does not allow any aggregation, i.e. every source transmits its own packet only. This scheme is implemented as a benchmark and if used together with the fixed schedule, it is actually equivalent to a single-hop WirelessHART, where the schedule is chosen once and no aggregation or relaying is performed by the nodes.

- Random aggregation scheme (RA), each source randomly and independently selects a packet to aggregate.

- Least overheard aggregation scheme (LO), in which each source node, during the whole network operation time, keeps track of the number of times it has overheard packets from other sources (either directly from the source itself or aggregated from another source), and selects the packet to aggregate that it has overheard the least number of times (ties are broken randomly). The rationale underlying this heuristic is the following: in the absence of any information about the channels between other sources and the controller, the number of times a packet from another source has been overheard is taken as a proxy for the "average channel quality" between the source and a generic receiver (including the coordinator). The least-overheard strategy aims to help the "worst" source in this sense.

The following three schemes assume the use of the adaptive schedule (as they make little sense otherwise):

- First-heard-first aggregation scheme (FHF), where each source node aggregates, from among the packets it has received, the packet coming from the source that had the earliest time slot in the current superframe. Since the adaptive schedule scheme starts with the source that has the worst channel to the controller, this scheme is likely to help nodes with higher probability of packet error.

- Fair least overheard aggregation scheme (FLO), works similar to the LO scheme, but to increase diversity, we regard it as advantageous if the controller hears a packet from as many distinct spatial sources as possible, and to increase that number, each aggregating node prefers sources from which it has received the packet directly. In other words, every node prioritizes the packets which were not aggregated by other sources in previous time slots within the current transmission round first and then looks at the number of times each of the packets was overheard. Again, ties are broken randomly. If none or all of the considered packets were already aggregated in the current superframe, the scheme works as the LO protocol.

- Genie aided aggregation scheme (GA), assumes that every source node has knowledge of the channel conditions between all nodes in the network and also knowledge of all packets which have been aggregated in the current superframe. At time slot $T_{k}$ the node $k$ which is assigned to transmit in slot $T_{k}$ has the knowledge of the transmissions done by nodes $0 \ldots(k-1)$ in time slots 
$T_{0} \ldots T_{k-1}$. First, node $k$ removes the packets which have already been aggregated at least once in the current round from its list of $n_{k}$ overheard packets. If all the packets in $n_{k}$ already have been forwarded in the current superframe, none of them are removed. Next, for each of the packets left in the list (if more than one packet left) node $k$ looks at the channel conditions between the originator of the packet and the nodes scheduled in the upcoming time slots $k \ldots(N-1)$. The packet having the least number of possible relayers with better channel conditions to the originator of the packet than the current node, is selected for aggregation. If several packets in the list of overheard packets have the same number of possible relayers, all with better channel conditions to the packet originator than node $k$, the actual channel PER differences are compared to choose the packet to aggregate. Following this scheme, each node will aggregate a packet which has the lowest chances to be aggregated by any of the following nodes in the schedule.

\section{RESULTS}

In this section, results from the performance evaluation are presented. To emulate a random node deployment, we generate a symmetric matrix containing packet error probabilities between all pairs of nodes. The matrix is static, randomly generated, and unknown to the source nodes and to the controller. Since no final conclusion about the exact parameters describing the channels encountered in industrial environments has been derived yet and to represent the worst case scenario, packet error rate values are chosen randomly from a uniform distribution within the open interval $(0,1)$. Additionally, this way of selecting the channel error probability values significantly reduces the required simulation time and does not affect the performance comparison of the evaluated protocols. For every simulated scenario, 150 different node deployment situations, i.e. channel matrix realizations, are used and 100000 transmission rounds are made for every such realization.

\section{A. Considered performance indicators}

Several different performance indicators are considered: success probability, i.e., the probability that all source packets are successfully delivered to the destination before their deadlines; the total number of erroneous packets within each superframe; individual source packet delivery rates (PDR); and finally the number of consecutive errors for each source from one superframe to the next. The reason for considering this set of performance indicators is that different applications have different requirements, and our suggested scheme is applicable to a range of different use cases. Success probability can be important for applications where every control decision is made based on sensor readings from several sensor nodes. In such cases it is crucial that all data packets are delivered to the controller before a common deadline, the time when the decision has to be made. The total number of erroneous packets within each superframe then indicates how far from success the system is. However, in many other applications, the set of sensors may have different sampling periods and be connected to different control functions, such that some errors can be tolerated by the system. Thus, individual source packet delivery rates and number of consecutive errors from every sensor are important performance indicators for such applications.

\section{B. Simulation results}

The performance of the protocols described above was evaluated via Matlab simulations. The simulator was verified against a numerical model. The developed model is based on a timehomogeneous discrete-time Markov chain, and has the success probability as its main performance indicator. The model describes the case with no feedback, a fixed schedule and random choice of the aggregated packets. The other schemes considered by the simulator were not easily amenable to mathematical modeling, but the fact that the majority of the program code underlying the simulation model for this simple scheme is shared with the simulation models for the other schemes, increases our confidence that the simulation results for these schemes are valid as well. A detailed description of the model and the comparison results are given in [19]. Evaluation shows that the numerical model and the simulator match very well.

\section{1) Comparison of four investigated schedules}

Once the simulator was checked against the mathematical model, it was used to evaluate the relaying and aggregation schemes suggested above. We simulate 150 different channel matrix realizations and run 100000 superframes for each of the realizations. First, different source transmission schedules were studied and compared. Fig. 1 shows the success probability averaged over all simulated channel realizations for $N=10$ source nodes. The bars are grouped according to schedules, and for every schedule the results for the scheme with no aggregation, NA, with random aggregation, RA and finally with the least overheard aggregation, LO, strategy are presented.

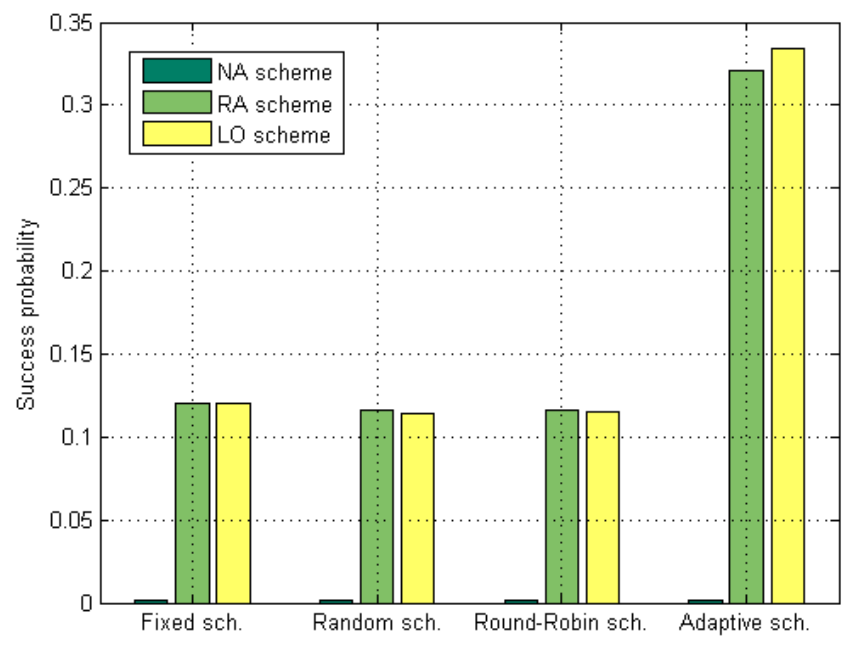

Fig. 1. Success probability for different source transmission schedules $(N=$ 10 source nodes, and aggregation using the NA, RA and the LO schemes)

First, looking at Fig. 1 it can be noticed how significantly the adaptive schedule outperforms all the other three. The adaptive schedule gives the best results as the transmission order generated by the scheduler is adapted to the channel conditions. The 
controller keeps track of the packets received from all source nodes and, based on this information, estimates the channel error rates. These estimations are used to adapt the schedule, such that after a number of transmission rounds, a new schedule is constructed by letting the source with the worst channel conditions transmit first, the source with the second worst channel second and so on. This gives more nodes a chance to overhear packets from the sources with bad channels to the controller, such that they can help by forwarding these packets to the destination. Simulations show that the schedule adapts quite fast and the transmission order changes only 138 times on average throughout 100,000 superframes. However, note that with the adaptive schedule, the source with the best channel to the controller, and thereby transmitting last, will never get any help from any other sources once the schedule has stabilized. In contrast, with the schemes using random or round-robin schedules, the node with the best channel to the destination can sometimes be aided by other nodes as well. Further, it can be seen from Fig. 1 that the results for random and round-robin schedules are very similar, since with relatively small number of source nodes, 10 in this example, and thus also quite few distinct scheduling options, it is very likely that these schemes will generate similar schedules quite often. Interestingly, it can be noticed that with the random and round-robin schedules, the RA scheme outperforms the LO scheme, and vice versa for the fixed and the adaptive schedules. The reason for this is that according to the LO scheme, a node selects a packet for aggregation based on the history of packets it has overheard in previous transmission rounds. If the schedule changes, the history cannot reflect the channel conditions as well as it does for the fixed or the adaptive schedule, as it takes a few superframes for the schedule to stabilize. Thus, it is better to randomly select a packet to aggregate than to base the decision on a parameter that is no longer valid. Finally, it can be seen that the resulting success probability for the NA scheme is equal for all the schedules since no aggregation is done according to this scheme and thus, the order in which source nodes perform their transmissions is irrelevant. This scheme represents the behaviour of a traditional TDMA network where one single packet is transmitted in each time slot and it is what we would have expected from a WirelessHART network if no retransmissions are scheduled.

However, it should be noted that the proposed schemes perform packet aggregation and thus achieve performance improvements at the cost of additional energy used for overhearing and sending longer aggregated packets. To be fair to traditional TDMA schemes, which would schedule one additional retransmission time slot for each of the sources, we simulate this case as well. When every source is allowed to retransmit its own packet once, the success probability is increased from 0.0013 to 0.0175 , which is still much lower than the performance achieved by any of the aggregation schemes, since apart from time diversity, they also benefit from spatial diversity and moreover, the schemes are adaptive and thus some of the packets can be retransmitted more than once.

Also, it is important to mention that the schedule comparison results presented in Fig. 1 remain true even in case of a weak feedback channel, Fig. 2. We have performed simulations for the extreme case of a completely lossy feedback channel, i.e. without any feedback, and the results show that as in Fig. 1 the adaptive schedule outperforms all other schedules significantly. For example, Fig. 2 shows that when the success probability levels for the random schedule coupled with the NA and LO schemes are equal to 0.0013 and 0.0110 , respectively, the success probability for the adaptive schedule combined with the LO scheme reaches 0.0471 . In the case of no feedback, the adaptive schedule coupled with the RA scheme outperforms the LO scheme. For example, the RA scheme coupled with the random and adaptive schedule results in 0.0169 and 0.0558 success probabilities respectively. This is because in the changing transmission schedule the RA scheme is fairer to all the sources and thus performs better when no feedback is available. On the other hand, when the schedule is stable, the LO scheme works better since the packets which were overheard the least number of times have the lowest chance to be delivered correctly to the controller. Moreover, it is noticeable that the improvement achieved by aggregation and schedule adaptation is decreased compared to the case with feedback present since many time slots are wasted on aggregating packets already successfully received at the destination instead of sending the missing ones.

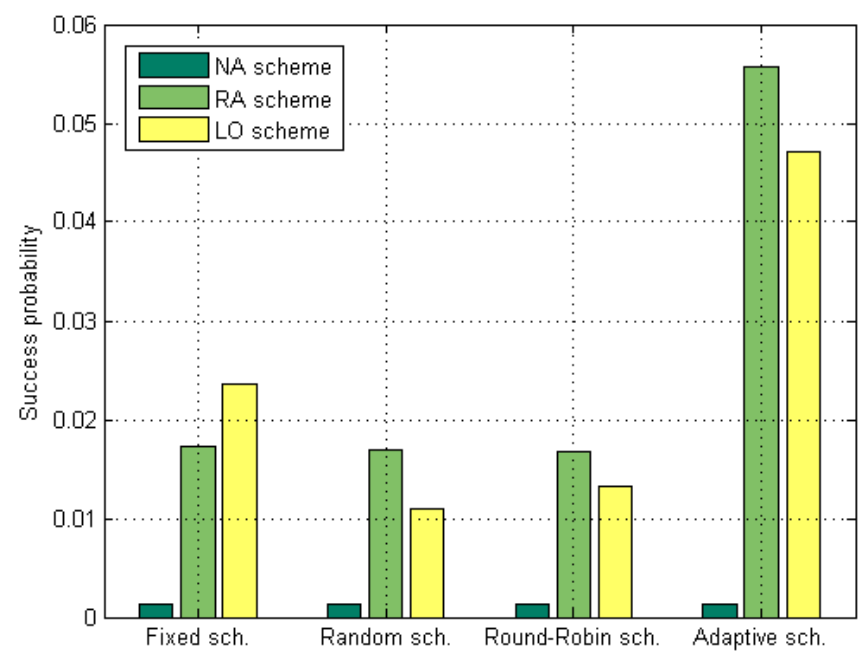

Fig. 2. Success probability for different source transmission schedules and schemes with no available feedback $(N=10$ source nodes, and aggregation using the NA, RA and the LO schemes)

Additionally, since industrial packet are often short and might have lower PER, simulations were performed for a more optimistic scenario with the channel PERs randomly selected from the open interval $(0,0.5)$. The results show that all the conclusions and trends derived from Fig. 1 remain valid, although the performance differences achieved by our proposed schemes are slightly less pronounced. For example, the success probability levels for the random schedule coupled with the NA and LO schemes are equal to 0.05 and 0.60 , respectively, whereas the success probability for the adaptive schedule and LO scheme reaches 0.87 . However, as stated in the beginning of this Section, in the remainder of this paper we will restrict our discussions to the less optimistic but more general case, where the PERs are drawn uniformly from $(0,1)$. 


\section{2) Comparison of the packet selection schemes}

As it can be seen from Fig. 1, the adaptive schedule leads to a considerable gain. Consequently, when comparing different packet selection schemes in Fig. 3, we only present results for the adaptive schedule. Five different packet selection schemes are compared: first-heard-first, FHF; random aggregation, RA; least overheard, LO; fare least overheard, FLO; and finally genie aided, GA. First, we notice that the FHF scheme results in the lowest success probability, since this scheme is the least fair scheme - even less fair than the RA scheme, helping almost exclusively the first few sources in the superframe. Next, it can be seen from Fig. 3 that both the LO and the FLO schemes work better than the RA scheme, since the least overheard property considers the number of times each packet has been overheard, and thus sources aim to prioritize packets from nodes having bad channels to most of other nodes. We also note that the FLO scheme performs better than the LO, since the fair property aims to help as many different sources per round as possible, thus increasing the chances for success. As expected, the best scheme among the studied ones is the GA scheme, since it assumes that all nodes have perfect channel knowledge and also knowledge of all packets sent in the current superframe. However, the improvement achieved with the GA and the FLO schemes is not significantly higher than that of the RA scheme and it becomes even smaller when the simulations are performed for the channel matrices with lower average PER values taken from $(0,0.5)$, since in the case when less source nodes require relaying it is highly likely that all the investigated packet selection schemes will choose the same packet for aggregation. Thus, depending on the application, it can be beneficial to use the RA scheme, given that the adaptive schedule is used, as it is less complex to implement.

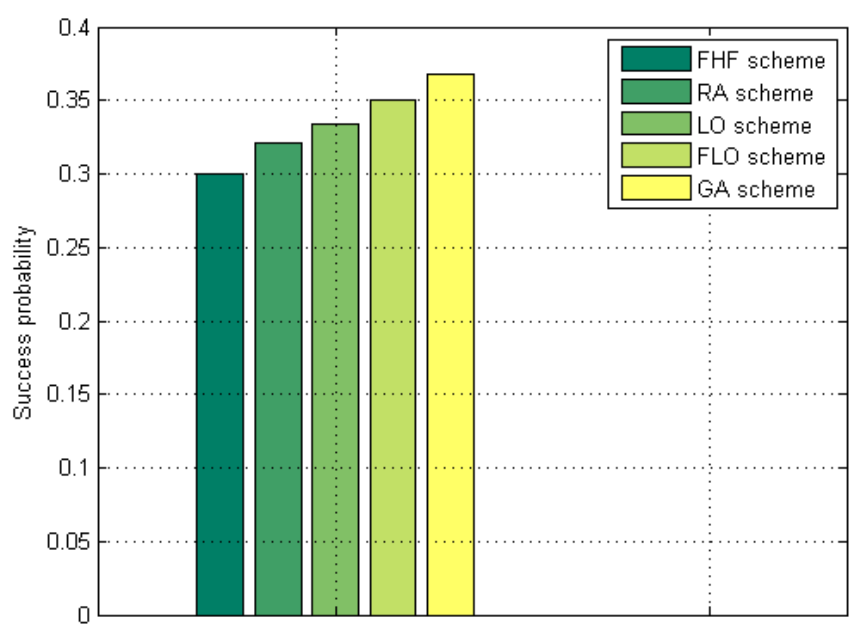

Fig. 3. Adaptive schedule: different packet selection schemes $(N=10)$

Looking at the PDR separately for each source, the performance of the investigated aggregation schemes can be compared even further. TABLE 1 shows the PDR averaged over all channel realizations for five selected nodes out of the ten source nodes. Nodes 0 and 1 are chosen as they have the worst and secondworst channel condition to the controller respectively, node $4-$ the fifth worst, whereas nodes 8 and 9 have the second best and the best channel conditions to the controller. The results are shown for the NA, FHF, FLO and GA schemes. The FLO and GA schemes are chosen as they have shown the best results in Fig. 3, the FHF protocol because it helps different nodes differently and the NA scheme is added as a reference. It can be seen from the table that the FHF scheme, leading to the worst success probability in Fig. 3, improves the performance of Node 0 and Node 1 significantly, but at the expense of the other source nodes. The FLO and the GA schemes, in contrast to the FHF, try to help all the sources equally and consequently show lower PDR for the first few sources, but higher for all the other nodes.

TABle 1. PDR FOR FIVE CHOSEN SOURCES (ADAPTIVE SCHEDULE, $N=10$ NODES).

\begin{tabular}{|c|c|c|c|c|c|}
\hline & Node 0 & Node 1 & Node 4 & Node 8 & Node 9 \\
\hline NA & 0.0853 & 0.1774 & 0.4449 & 0.8138 & 0.9159 \\
\hline FHF & 0.9954 & 0.9337 & 0.7320 & 0.8298 & 0.9159 \\
\hline FLO & 0.6880 & 0.7728 & 0.8503 & 0.8746 & 0.9160 \\
\hline GA & 0.7666 & 0.7664 & 0.8333 & 0.8857 & 0.9161 \\
\hline
\end{tabular}

Although the success probability is very important whenever all the source data must be delivered to the controller in time, it is still interesting and informative to know how many source packets are lost in each superframe. TABLE 2 shows for the different aggregation schemes the percentage of superframes that resulted in $0,1,2, \ldots$ or 10 erroneous packets at the controller; the cases with a particular number of erroneous packets in a superframe reaching $20 \%$ are marked in bold.

TAble 2. Percentage of $0,1,2, \ldots$ OR 10 erroneous PaCKets in A SUPERFRAME (ADAPTIVE SCHEDULE, $N=10$ SOURCE NODES).

\begin{tabular}{|c|c|c|c|c|c|}
\hline \multirow{2}{*}{} & \multirow{2}{*}{ NA } & \multicolumn{4}{|c|}{ Adaptive schedule } \\
\cline { 3 - 6 } & & FHF & RA & FLO & GA \\
\hline 0 & 0.13 & $\mathbf{2 9 . 9 4}$ & $\mathbf{3 2 . 0 9}$ & $\mathbf{3 5 . 0 3}$ & $\mathbf{3 6 . 8 1}$ \\
\hline 1 & 0.98 & $\mathbf{2 2 . 7 3}$ & $\mathbf{2 1 . 8 7}$ & $\mathbf{2 0 . 2 7}$ & 18.98 \\
\hline 2 & 4.09 & $\mathbf{2 0 . 1 1}$ & $\mathbf{2 0 . 0 7}$ & 19.85 & 19.68 \\
\hline 3 & 11.09 & 8.46 & 7.45 & 6.49 & 6.19 \\
\hline 4 & $\mathbf{2 0 . 1 3}$ & 11.18 & 11.19 & 11.19 & 11.19 \\
\hline 5 & $\mathbf{2 5 . 0 1}$ & 2.11 & 1.88 & 1.73 & 1.71 \\
\hline 6 & $\mathbf{2 1 . 1 3}$ & 4.17 & 4.18 & 4.18 & 4.18 \\
\hline 7 & 12.11 & 0.32 & 0.30 & 0.29 & 0.29 \\
\hline 8 & 4.37 & 0.88 & 0.88 & 0.88 & 0.88 \\
\hline 9 & 0.89 & 0.02 & 0.02 & 0.02 & 0.02 \\
\hline 10 & 0.07 & 0.07 & 0.07 & 0.07 & 0.07 \\
\hline
\end{tabular}

It can be seen from the table that when no aggregation is done, four, five or even six packets miss their deadlines in the majority of all superframes. Introducing packet aggregation and the adaptive schedule improves the numbers dramatically, i.e. in the majority of cases only one or two packets per superframe do not arrive at the destination correctly. However, in $0.07 \%$ of all the investigated cases, all source packets arrive to the destination 
with errors and no improvement is made by any of the aggregation schemes, and thus performance is similar to the NA scheme. This is the drawback of having only source nodes forwarding each other's packets and not allowing retransmissions or additional nodes serving only as relayers.

Another important performance indicator for industrial applications is the number of times each scheme encounters two or three consecutive errors from a particular source. This measure is of great importance for industrial systems which often can tolerate one packet error from a source, but have to turn machines into a safe state when two consecutive errors occur and actually switch off in case of three consecutive errors. TABLE 3 shows the total number of errors and the number of two and three (marked in bold) consecutive errors, as a percentage of all the simulated superframes. The numbers are averaged over all channel realizations and, as in TABLE 1, presented for source nodes $0,1,4,8$ and 9. It can be seen from the table that introduction of packet aggregation and the adaptation of the schedule to the channel conditions significantly reduces the number of two and three consecutive errors.

TABle 3. Percentage of CONSECUTIVE ERRORS FOR A SELECTED SUbSET OF ALL SOURCE NODES (ADAPTIVE SCHEDULE, $N=10$ SOURCE NODES)

\begin{tabular}{|c|c|c|c|c|c|c|}
\hline & & $\begin{array}{c}\text { Node } \\
0\end{array}$ & $\begin{array}{c}\text { Node } \\
1\end{array}$ & $\begin{array}{c}\text { Node } \\
4\end{array}$ & $\begin{array}{c}\text { Node } \\
8\end{array}$ & $\begin{array}{c}\text { Node } \\
9\end{array}$ \\
\hline \multirow{4}{*}{ NA } & Total & 91.47 & 82.26 & 55.51 & 18.62 & 8.41 \\
\cline { 2 - 7 } & 2 & 30.34 & 26.95 & 16.55 & 3.33 & 1.03 \\
\cline { 2 - 7 } & $\mathbf{3}$ & $\mathbf{2 7 . 9 8}$ & $\mathbf{2 2 . 6 8}$ & $\mathbf{1 0 . 0 8}$ & $\mathbf{0 . 9 0}$ & $\mathbf{0 . 2 0}$ \\
\hline \multirow{4}{*}{ FHF } & Total & 0.46 & 6.63 & 26.80 & 17.02 & 8.41 \\
\cline { 2 - 7 } & 2 & 0.02 & 0.75 & 6.08 & 2.92 & 1.03 \\
\cline { 2 - 7 } & $\mathbf{3}$ & $\mathbf{0 . 0 0}$ & $\mathbf{0 . 1 6}$ & $\mathbf{2 . 3 9}$ & $\mathbf{0 . 7 6}$ & $\mathbf{0 . 2 0}$ \\
\hline \multirow{4}{*}{ FLO } & Total & 31.20 & 22.72 & 14.97 & 12.54 & 8.40 \\
\cline { 2 - 7 } & 2 & 7.56 & 4.68 & 2.40 & 1.77 & 1.03 \\
\cline { 2 - 7 } & $\mathbf{3}$ & $\mathbf{3 . 3 6}$ & $\mathbf{1 . 6 3}$ & $\mathbf{0 . 6 3}$ & $\mathbf{0 . 3 7}$ & $\mathbf{0 . 2 0}$ \\
\hline \multirow{3}{*}{ GA } & Total & 23.34 & 23.36 & 16.67 & 11.43 & 8.39 \\
\cline { 2 - 7 } & 2 & 4.93 & 4.93 & 2.85 & 1.57 & 1.03 \\
\cline { 2 - 7 } & $\mathbf{3}$ & $\mathbf{1 . 8 0}$ & $\mathbf{1 . 8 3}$ & $\mathbf{0 . 7 7}$ & $\mathbf{0 . 3 3}$ & $\mathbf{0 . 2 0}$ \\
\hline
\end{tabular}

\section{3) Results for different numbers of source nodes}

Next, we look how a change in the number of the source nodes affects the results presented above. First, Fig. 4 shows the results for different aggregation schemes using the adaptive schedule only (since it has the best performance among the compared schedules as it will be shown later), and $N=5,10$ and 20 source nodes. In all three cases, each source node has only one allocated time slot; the deadline for packet delivery is the same for all the nodes and equals the end of the superframe. Consequently, the length of each superframe is shorter for five source nodes than for ten. It can be seen that all the trends observed in Fig. 3 for $N=10$ are preserved also for $N=5$ and 20. Similarly, it is interesting to see that the success probability increases when the number of source nodes grows. This is due to the longer superframes and the increased number of possible relayers for every node.

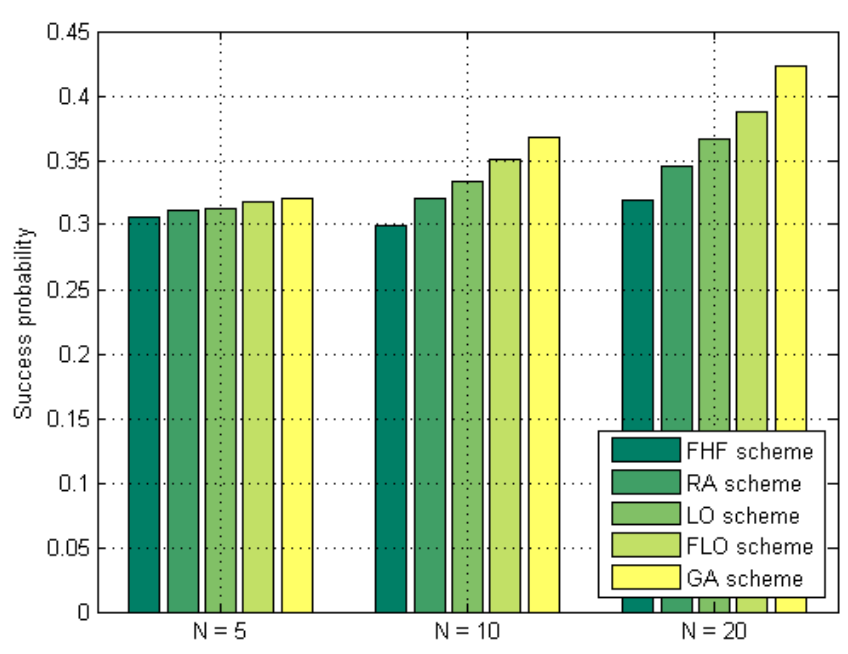

Fig. 4. Success probability comparison: adaptive schedule, $N=5,10$ and 20 source nodes (FHF, RA, LO, FLO and GA schemes)

Fig. 5 shows the results for different packet transmission schedules, all using the LO aggregation scheme, and presented for three sets of source nodes, i.e., $N=5,10$ and 20. It can be seen from the figure that when the number of source nodes increases, the success probability for the fixed, random and roundrobin schedules reduces, while it instead improves when the adaptive schedule is used. For the fixed, random and round-robin schedules, success probability decreases since with a higher number of sources, more and more packets have to be correctly delivered. The schedules are not flexible enough to take advantage of the higher spatial diversity implied with the larger number of possible relay nodes available. The adaptive schedule, on the other hand, organizes the transmission order in a way that more sources can help each other by forwarding the data.

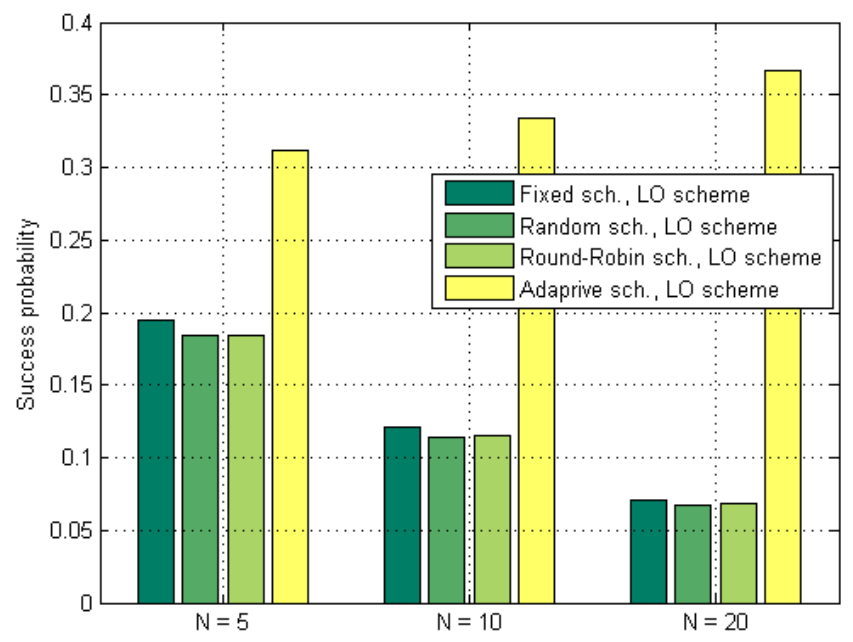

Fig. 5. Success probability comparison: LO scheme, $N=5,10$ and 20 source nodes (fixed, random, round-robin and adaptive schedules)

Interestingly, while the success probability improves with the increased number of source nodes only in the case of the adaptive schedule, the PDR for each separate node is growing as the number of nodes increases for all the investigated schemes. 
As an example, Fig. 6 and Fig. 7 show the PDR averaged over all channel realizations for five source nodes, with the LO aggregation scheme and the adaptive and random schedules respectively. The five source nodes selected for presentation in Fig. 6 and Fig. 7 are present in all three simulations, i.e., in $N=$ 5 only five source nodes exists, while in the simulations for $N=$ 10 and 20, the same five source nodes are subsets of the 10 and 20 nodes respectively.

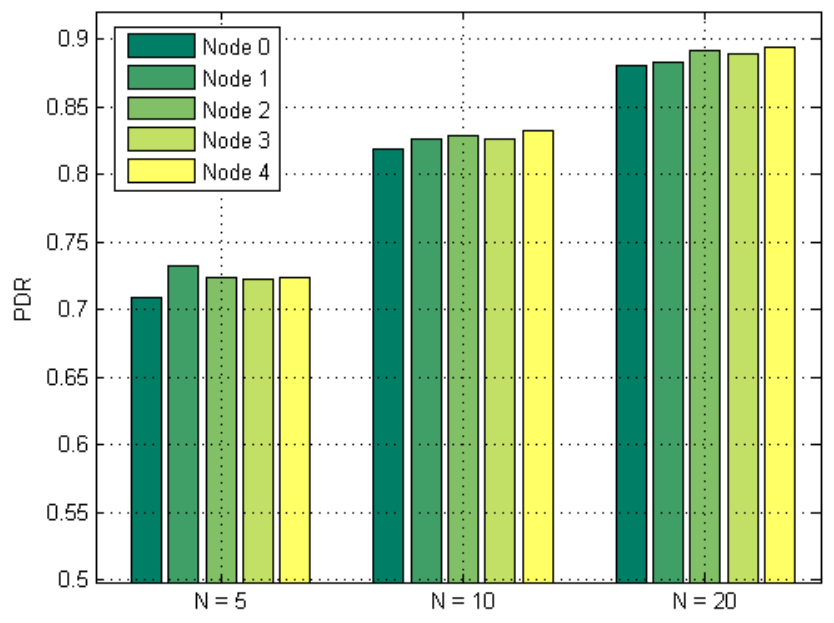

Fig. 6. Adaptive schedule: PDR comparison for $N=5,10$ and 20 source nodes (LO scheme, all five source nodes are present in all three simulations)

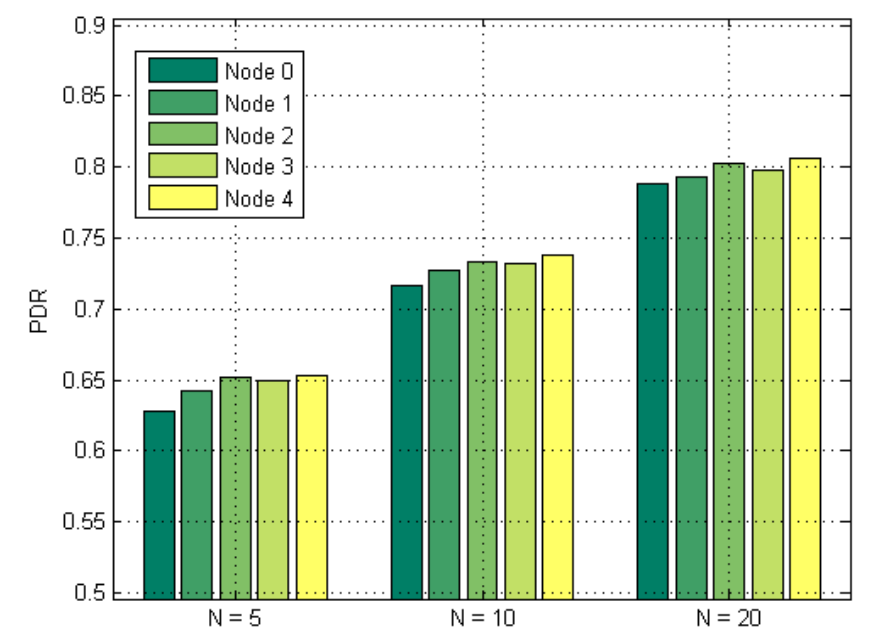

Fig. 7. Random schedule: PDR comparison for $N=5,10$ and 20 source nodes (LO scheme, all five source nodes are present in all three simulations)

The PDR is improved with a higher number of source nodes in both cases and this improvement can be explained by the fact that with more nodes the system has more possible relayers for each source. Also, it is interesting to see that for the random schedule, Fig. 7, the relative PDR differences for the five sources stay the same for all studied numbers of sensors, which is not the case for adaptive schedule, Fig. 6 . This is connected to the adaptive transmission order change, i.e. after a number of superframes, the schedule stabilizes and the nodes transmitting close to the end of the superframe receive less help from aggregation. When the number of source nodes grows, some of the nodes, having relatively bad channels, which transmitted in the beginning of the superframe for $N=5$ are being pushed towards the end of the superframe when $N=10$ and 20 and therefore receiving less help.

\section{Discussion}

Looking at all the results above, it is clear that our proposed schemes with scheduling and aggregation improve the performance in terms of all the considered performance indicators. Section IV.B.1 presents the results for the performance comparison of source relaying and packet aggregation with classical TDMA schemes allocating one or two time slots for transmission and retransmission, respectively, for each of the source nodes. In both cases, source relaying with packet aggregation is beneficial due to exploitation of spatial diversity and flexibility given to the sources when choosing which packet to aggregate. Evaluation of different performance indicators shows that the final choice of which algorithm to use must be based on the most important performance measure for a particular application. For example, when schedule adaptation is not possible, packet aggregation and source relaying significantly outperform TDMA schemes allowing one retransmission from each source even when using a fixed schedule. Adaptation of the schedule improves the reliability dramatically regardless of the quality of the feedback channel and the channel PER. Even further improvements can be achieved by carefully choosing the selection strategy for the aggregated packets. The FLO scheme performs best, second only to the artificial GA protocol, for all the investigated performance indicators. However, if computational resources are limited or if no feedback is available in the system, the RA scheme should be chosen. The FLO scheme is fair and tries to help the maximum number of nodes possible in a superframe, and should be selected when fairness is important. However, if one node has higher priority than the others, FHF scheme should instead be used. Increasing the number of source nodes improves the PDR for all the sources, as this also increases the superframe length and hence implicitly also the number of retransmission attempt by aggregation. However, if success probability is of greater importance than PDR, an increased number of nodes leads to an improvement for the adaptive schedule only. Thus, if schedule adaptation is not possible and success probability is the key parameter, the same level of success probability can be achieved only with a reduced, compared to networks using the adaptive schedule, number of source nodes.

\section{CONCLUSIONS}

Reliability and timeliness are two main requirements in IWSNs, which must be fulfilled before the transition between currently used wired and much cheaper and simpler wireless networks becomes possible. One of the much discussed ways to improve reliability of wireless systems suffering from errors due to harsh channel conditions in industrial environments, is adaption of cooperative communication or relaying. The main goal of this paper was to evaluate the performance gain of source-based relaying schemes with packet aggregation implemented in all source nodes and to find the scheduling algorithm which maximizes the gain. The results show that allowing source nodes to perform relaying together with aggregation and adjusting the 
schedule based on the currently available channel estimations yields significant performance improvements. Aggregation using carefully chosen packet selection schemes improves the performance further.

Even further gains are likely to be achieved by e.g. combining packet aggregation and network coding. These schemes are the subject for possible future work. Additionally, the current work can be extended by taking into consideration networks with different packet deadlines, studying the system performance in environments with changing channel conditions and evaluating scheduling and relaying opportunities for nodes working on several wireless channels in parallel and thus having a chance to listen to only a subset of the transmitted packets due to concurrent transmissions on different frequency channels.

\section{REFERENCES}

[1] D. Chen, M. Nixon, and A. M. Mok, Wireless HART: Real-Time Mesh Network for Industrial Automation: Springer Science+Business Media, 2010.

[2] HART Communication Foundation, TDMA Data Link Layer Specification, HCF SPEC 075 Revision 1.1, 17 May, 2008.

[3] Chinese Industrial Wireless Alliance, $\mathrm{http} / / / \mathrm{www}$. industrialwireless.cn/en/index.asp.

[4] American National Standards Institute, Washington DC., ANSI/ISA100.11a-2011 - Wireless systems for industrial automation: Process control and related applications, 2011.

[5] IEEE Standards Association, "IEEE Standard for local and metropolitan area networks," PART 15.4: Low rate Wireless Personal Area Networks (LR-WPANs) Amendment 1: MAC sublayer, IEEE, 2012.

[6] V. Ç. Güngör, and G. P. Hancke, Industrial Wireless Sensor Networks: Applications, Protocols, and Standards, Boca Raton: CRC Press Taylor\&Francis Group, 2013.

[7] A. Willig, "Recent and Emerging Topics in Wireless Industrial Communications: A Selection," IEEE Trans. Ind. Inf., vol. 4, no. 2, pp. 102-124, 2008.

[8] S. Petersen, and S. Carlsen, "WirelessHart versus ISA100.11a: The Format War Hits the Factory Floor" IEEE Ind. Elec. Mag., vol. 5, no. 4, pp. 23-34, 2011.

[9] J. Song, S. Han, A. M. Mok et al., "WirelessHART: applying wireless technology in real-time industrial process control," Proc.
IEEE Real-Time and Embedded Tech. and App. Symp., St. Louis, Missouri, USA Apr. 2008, pp. 377-386.

[10] S. Han, X. Zhu, A. M. Mok et al., "Control over WirelessHART Network," Proc. Annual Conf. on IEEE Ind. Electronics Society Glendale, AZ, USA, Nov. 2010, pp. 2114-2119.

[11] S. N. Diggavi, N. Al-Dhahir, A. Stamoulis et al., "Great Expectations: The Value of Spatial Diversity in Wireless Networks," Proc. of the IEEE, vol. 92, no. 2, pp. 219-270, 2004.

[12] X. S. Shen, A. Hjrungnes, Q. Zhang et al., "Special issue on cooperative networking - challenges and applications (part 1)," IEEE Journal on Selected Areas in Communic., vol. 30, no. 2, pp. 241-244, 2012.

[13] A. Willig, and E. Uhlemann, "Deadline-Aware Scheduling of Cooperative Relayers in TDMA-Based Wireless Industrial Networks," Wireless Networks, vol. 20, no. 1, pp. 73-88, 2014.

[14] S. Girs, E. Uhlemann, and M. Björkman, "The effects of relay behavior and position in wireless industrial networks," Proc. IEEE Int. Workshop Factory Commun. Syst., Lemgo, Germany, May 2012, pp. 183-190.

[15] S. Girs, E. Uhlemann, and M. Björkman, "Increased reliability or reduced delay in wireless industrial networks using relaying and Luby codes," Proc. IEEE Int. Conf. on Emerging Tech. and Factory Autom., Cagliari, Italy, Sept. 2013, pp. 1-9.

[16] A. Ulusoy, O. Gurbuz, and A. Onat, "Wireless Model-Based Predictive Networked Control System Over Cooperative Wireless Network" IEEE Trans. Ind. Inf., vol. 7, no. 1, pp. 41$51,2011$.

[17] J. Neander, T. Lennvall, and M. Gidlund, "Prolonging wireless HART network lifetime using packet aggregation," Proc. IEEE Int. Symp. Ind. Elec., Gdansk, Poland Jun. 2011, pp. 1230 - 1236.

[18] M. Baldi, R. Giacomelli, and G. Marchetto, "Time-Driven Access and Forwarding for Industrial Wireless Multihop Networks," IEEE Trans. Ind. Inf., vol. 5, no. 2, pp. 99 - 112, 2009.

[19] This reference is blinded for reasons of anonymity.

[20] S. Girs, A. Willig, E. Uhlemann et al., "On the role of feedback for industrial wireless networks using relaying and packet aggregation," Proc. IEEE Int. Conf. on Ind. Tech., Busan, South Korea, Feb. 2014, pp. 743-748.

[21] B. Rong, and A. Ephremides, "Cooperative Access in Wireless Networks: Stable Throughput and Delay," IEEE Trans. on Information Theory, vol. 58, no. 9, pp. 5890-5907, 2012. 